\title{
A Stable $Q$ Compensated Reverse Time Migration Method Based on Excitation Amplitude Imaging Condition
}

\author{
Qingqing $\mathrm{Li}^{1, *}$, Li-Yun $\mathrm{Fu}^{1}$, Weijia Sun ${ }^{2}$, Wei $\mathrm{Wei}^{2}$ and \\ Wanting $\mathrm{Hou}^{1}$ \\ ${ }^{1}$ School of Geosciences, China University of Petroleum, Shandong 266555, China. \\ 2 Institute of Geology and Geophysics, Chinese Academy of Sciences, Beijing 100029, \\ China.
}

Received 26 March 2018; Accepted (in revised version) 25 March 2019

\begin{abstract}
The stability and efficiency, especially the stability, are generally concerned issues in $Q$ compensated reverse time migration $(Q-R T M)$. The instability occurs because of the exponentially boosted high frequency ambient noise during the forward or backward seismic wavefield propagation. The regularization and low-pass filtering methods are two effective strategies to control the instability of the wave propagation in Q-RTM. However, the regularization parameters are determined experimentally, and the wavefield cannot be recovered accurately. The low-pass filtering method cannot balance the selection of cutoff frequency for varying $Q$ values, and may damage the effective signals, especially when the signal-to-noise ratio (SNR) of the seismic data is low, the Q-RTM will be a highly unstable process. In order to achieve the purpose of stability, the selection of cutoff frequency will be small enough, which can cause great damage to the effective high frequency signals. In this paper, we present a stable Q-RTM algorithm based on the excitation amplitude imaging condition, which can compensate both the amplitude attenuation and phase dispersion. Unlike the existing Q-RTM algorithms enlarging the amplitude, the exponentially attenuated seismic wavefield will be used during both the forward and backward wavefield propagation of $Q$-RTM. Therefore, the new $Q$-RTM algorithm is relative stable, even for the low SNR seismic data. In order to show the accuracy and stability of our stable Q-RTM algorithm clearly, an example based on Graben model will be illustrated. Then, a realistic BP gas chimney model further demonstrates that the proposed method enjoys good stability and anti-noise performance compared with the traditional Q-RTM with amplitude amplification. Compare the $Q$-RTM images of these two models to the reference images obtained by the acoustic RTM with acoustic seismic data, the new Q-RTM results match the reference images quite well. The proposed method is also tested using a field seismic data, the result shows the effectiveness of our proposed method.
\end{abstract}

*Corresponding author. Email addresses: jyliqingqing@163.com (Q. Li), lfu@upc.edu.cn (L.-Y. Fu), swj@mail.iggcas.ac.cn (W. Sun), weiwei@mail.iggcas.ac.cn (W. Wei) hwtupc@163.com (W. Hou) 
AMS subject classifications: $86-08$

Key words: Stable, reverse time migration, attenuation.

\section{Introduction}

High-resolution seismic data processing is necessary for the detailed description of oil and gas reservoirs. In this paper, we discuss one resolution enhancement technique, which is the $Q$ compensated reverse time migration ( $Q$-RTM). When a seismic wave propagates in viscous media, especially the gas-bearing strata, the fundamental properties of the wavefield are changed with energy attenuation and phase dispersion [1]. As a result, the energy reflected from the deep layers below the gas-bearing layers is reduced, and the events are with a low resolution in the conventional migration images. The worst thing is that the imaged position of the interface is distorted because of the phase dispersion. It is necessary to compensate for these viscous effects in order to obtain the migration images with high fidelity.

Anelasticity and inhomogeneity of the subsurface weaken the high frequency seismic energy, which decrease seismic amplitudes and cause the phase dispersion. These effects can be described as the earth $Q$ filtering, defined in terms of a specified $Q$ model of the earth (Futterman, 1962; Strick, 1967, 1970). In order to compensate these absorption and dispersion in seismic data processing, various schemes have been developed. Among these schemes, the inverse $Q$ filtering method has been widely applied because of its effectiveness and high efficiency $[2,20]$. Nevertheless, the inverse $Q$ filtering method compensates the amplitude attenuation and phase dispersion along the depth direction trace by trace, while ignores the viscous effects in the horizontal direction. In fact, the viscous effects occur along the way of wavefield propagation, we should compensate the $Q$ effects along the real paths. It is obvious that the seismic data after inverse $Q$ filtering cannot be used to the subsequent amplitude versus offset (AVO) or amplitude versus angle (AVA) analysis due to the incorrectness of relative amplitude. Considering the weakness of the inverse $Q$ filtering method, Li et al. adopted a two-step scheme to approximate the paths of the wave in order to compensate the amplitude attenuation and phase dispersion in both depth and horizontal directions [13].

A plenty of prestack depth migration studies based on one way wave equation have been conducted to compensate the viscous effects for the down-going and up-going waves in the frequency domain $[5,15,16,23,25,27]$. These prestack depth migration schemes commonly compensate the attenuation using the imaginary part of a complex phase velocity. Although these schemes may be suitable for compensation, the one way wave equation migration is not suitable for the high dip structures.

The reverse time migration (RTM), which is based on two-way wave equation, has been considered as the most accurate migration technique for complex geological structures [10]. For Q-RTM algorithm, there are two kinds of viscoacoustic wave equations, 
i.e., the amplitude attenuation term and phase dispersion term coupled or decoupled equations. Based on the standard linear solid model (SLS), Deng and McMechan derived an amplitude attenuation and phase dispersion coupled viscoacoustic wave equation, and implemented the Q-RTM algorithm [6,7]. However, in $Q-R T M$, the true algorithm is to change the sign of the amplitude attenuation term, and keep the sign of the phase dispersion term [31]. Thus, the Q-RTM algorithms based on the coupled wave equation can only compensate the amplitude attenuation, and cannot obtain the accurate positions of the events, because the phase dispersion cannot be compensated.

Zhang et al. [28] and Zhu et al. [31] proposed their own fractional viscoacoustic wave equations with the amplitude attenuation and phase dispersion terms decoupled through different ways. Li et al. [14] improved the applicability of these equations for small $Q$ values. Based on these equations, plenty of researches on $Q-R T M$ algorithm $[14,31]$ and its computation efficiency $[3,14,18]$ have been conducted.

For all the above $Q$ compensated algorithms, the energy of seismic wave is amplified with exponential growth, the instability of the algorithms is inevitable. One effective method to suppress the instability is the low-pass filtering [14, 20,31], which can control the instabilities during the wave propagation. However, the cutoff frequency of the low-pass filtering method can damage the effective signals, especially the high-frequency components. In addition, the cutoff frequency is difficult to choose to balance the reflections of shallow and deep layers. Another method to overcome the instability is the regularization $[22,24,28]$, which is effective by adding one or more higher order regularization terms on the viscoacoustic wave equations in time-space domain. But the regularization parameters are usually empirical values, and the wavefield cannot be accurately recovered during the backward wavefield propagation. Although the $Q$ compensated leastsquares reverse time migration algorithm is stable $[9,19]$, the huge computation amount limits its application in seismic data processing.

Nguyen and McMechan proposed the excitation amplitude imaging condition in RTM to improves computational and storage efficiency [17]. With this method, only the maximum amplitude of source wavefield at each grid point and the corresponding time step need to be saved, which can solve the problem of great memory requirement. Gu et al. used the modified shortest-path method to overcome the distortion in the images generated with the excitation amplitude imaging condition [11]. Du et al. [8] and Zhou and Wang [29] applied the excitation amplitude imaging condition to the elastic RTM, solving the polarization reversal in PS wave images. Although the excitation amplitude imaging condition can avoid the great memory requirement, it faces the problem of lower stacked amplitudes, especially where there are multipath contributions. Zhang et al. adopted the excitation amplitude imaging condition to attenuate the crosstalk in the imaging of simultaneous-source data [26].

In this paper, we propose a theoretical framework of a stable $Q$-RTM algorithm, which can compensate both the amplitude attenuation and phase dispersion. In the previous $Q$-RTMs, we just change the sign of the amplitude attenuation term and keep the sign of the phase dispersion term in both forward and backward wave propagation, the en- 
ergy of the wavefield is exponentially amplified. However, in this paper, based on the excitation amplitude imaging condition, the wavefields are all exponentially attenuated in both forward and backward wavefield extrapolation of $Q$-RTM. Thus, our method has a better anti-noise performance than the previous $Q$-RTM method. By using this $Q$-RTM algorithm, both the amplitude attenuation and phase dispersion will be compensated, and the algorithm is stable.

The rest of this paper is organized as follows. First, we describe the conceptual theory of $Q$-RTM. Second, we introduce the formulations of the nearly constant- $Q$ viscoacoustic wave equation for extrapolating the source and receiver wavefields in attenuating media. Third, we present the numerical implementation of our stable $Q$-RTM. Finally, we demonstrate the effectiveness of our Q-RTM approach using synthetic seismic data from a graben model, the realistic BP gas model and a field seismic data.

\section{Theory of $Q$-RTM}

\subsection{Crosscorrelation imaging condition}

$Q$-RTM consists of three steps, the forward propagation of a wavefield from a source, the backward propagation of a wavefield from the receiver location, and the application of an imaging condition, for example, the zero-lag crosscorrelation imaging condition [4]:

$$
I(\mathbf{x})=\int_{0}^{T} S(\mathbf{x}, t) R(\mathbf{x}, t) d t
$$

where $S(\mathbf{x}, t)$ and $R(\mathbf{x}, t)$ are the forward propagated source wavefield and backwardpropagated receiver wavefields at time $t$ in a nonattenuating medium.

In an attenuating media, [31] had discussed the zero-lag crosscorrelation imaging condition in detail

$$
I^{C}(\mathbf{x})=\int_{0}^{T} S^{C}(\mathbf{x}, t) R^{C}(\mathbf{x}, t) d t
$$

where $I^{C}(\mathbf{x})$ is the migration image, $S^{C}(\mathbf{x}, t)$ and $R^{C}(\mathbf{x}, t)$ are the compensated forward and backward propagated wavefield in the viscous media at time $t$, and $T$ is the time length of the data. Fig. 1 schematically shows the propagation of wavefields for forward modeling and RTM. To illustrate the viscous effects, as shown in Fig. 1(b), we represent the attenuation from the source to the reflector by the exponential term $e^{-\alpha L_{F}}$ and the attenuation from the reflector to the receiver by $e^{-\alpha L_{B}}$, where $\alpha$ is the attenuation coefficient associated with the wavenumber $\mathbf{k}, L_{F}$ and $L_{B}$ are the forward and backward propagation paths, respectively. The amplitudes of the forward and backward wavefields should be amplified, thus, in Q-RTM, Eq. (2.2) can be rewritten as

$$
I^{C}(\mathbf{x})=\int_{0}^{T} S(\mathbf{x}, t) \underbrace{R_{V}(\mathbf{x}, t) e^{+\alpha L_{F}} e^{+\alpha L_{B}}}_{R(\mathbf{x}, t)} d t
$$




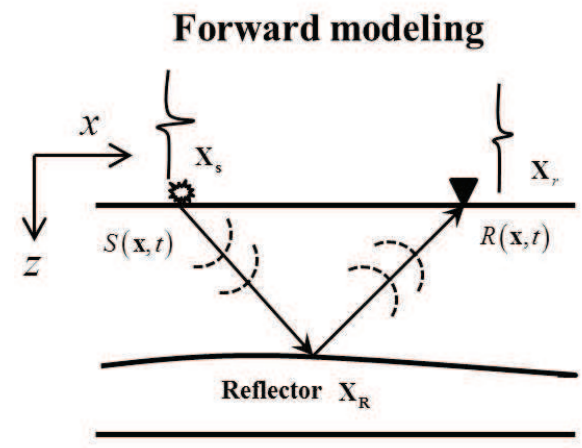

(a)

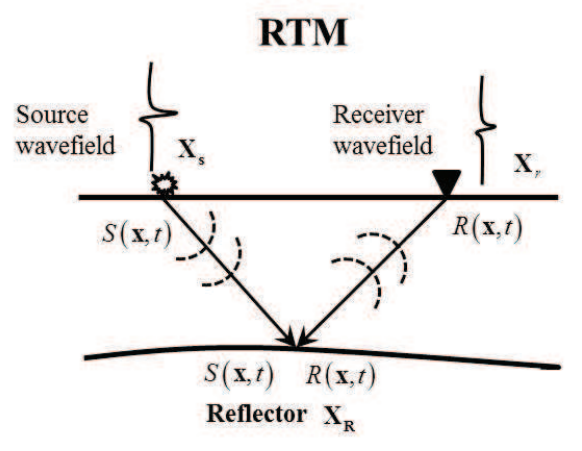

Forward modeling

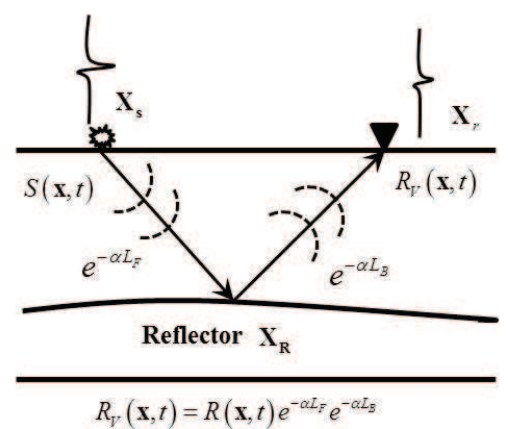

(b)

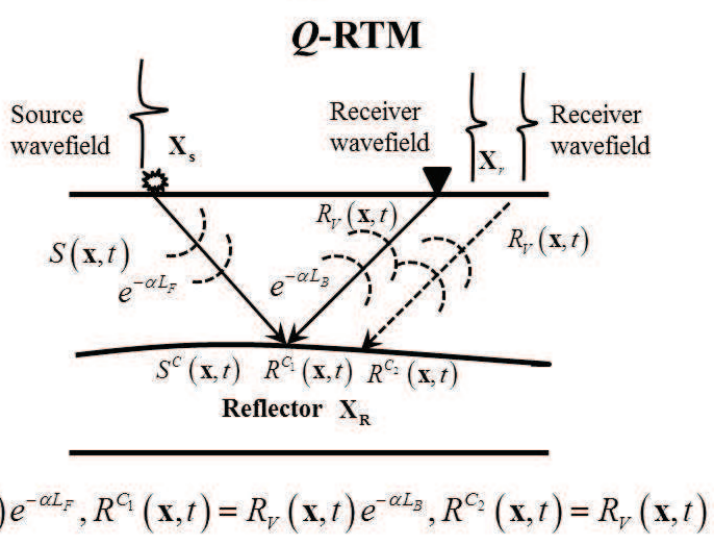

(d)

Figure 1: The schematic paths of (a) forward modeling and (c) RTM wavefield propagation in nonattenuation media, (b) the forward modeling and (d) stable Q-RTM wavefield propagation in attenuation media. The receiver recording in viscous media $R_{V}(\mathbf{x}, t)$ will be attenuated by $e^{-\alpha L_{F}}$ and $e^{-\alpha L_{B}}$ compared to the acoustic case. In our stable $Q$-RTM algorithm, the compensated source wavefield $S^{C}(\mathbf{x}, t)=S(\mathbf{x}, t) e^{-\alpha L_{F}}$ at the reflector point, the receiver wavefields $R^{C_{1}}(\mathbf{x}, t)=R_{V}(\mathbf{x}, t) e^{-\alpha L_{B}}$ and $R^{C_{2}}(\mathbf{x}, t)=R_{V}(\mathbf{x}, t)$ are all needed.

where $S(\mathbf{x}, t)$ and $R(\mathbf{x}, t)$ are the forward and backward propagated wavefields in the acoustic media, and $R_{V}(\mathbf{x}, t)$ represents the receiver wavefield with attenuation. However, the exponential terms of $e^{+\alpha L_{F}}$ and $e^{+\alpha L_{B}}$ can cause instability in $Q-R T M$.

We usually adopt a low-pass filtering or regularization methods to control the instability caused by the exponential terms of $e^{+\alpha L_{F}}$ and $e^{+\alpha L_{B}}$. Here, we define the cutoff wavenumber as $\mathbf{k}_{q}$, then the compensation operator with low-pass filtering method $\Lambda(\mathbf{k})$ can be expressed as

$$
\Lambda(\mathbf{k})= \begin{cases}e^{+\alpha(\mathbf{k}) L_{F}}\left(\text { forward), or } e^{+\alpha(\mathbf{k}) L_{B}}\right. \text { (backward), } & \mathbf{k} \leq \mathbf{k}_{q}, \\ e^{+\alpha\left(\mathbf{k}_{q}\right) L_{F}} \text { (forward), or } e^{+\alpha\left(\mathbf{k}_{q}\right) L_{B}} \text { (backward), } & \mathbf{k}>\mathbf{k}_{q},\end{cases}
$$


or

$$
\Lambda(\mathbf{k})= \begin{cases}e^{+\alpha(\mathbf{k}) L_{F}}(\text { forward }), \text { or } e^{+\alpha(\mathbf{k}) L_{B}} \text { (backward), } & \mathbf{k} \leq \mathbf{k}_{q} \\ 0, & \mathbf{k}>\mathbf{k}_{q} .\end{cases}
$$

According to Eqs. (2.4) and (2.5), we can see clearly that the high wavenumber effective signal cannot be compensated accurately or lost because of the cutoff wavenumber $\mathbf{k}_{q}$. Thus, the low-pass filtering method is unreliable. Except the low-pass filtering method, [21] proposed a regularization method for seismic resolution enhancement by utilizing an amplitude-compensated operator with stabilization in frequency domain, which can be expressed as

$$
\Lambda(\omega)=\frac{\beta(\omega)}{\beta^{2}(\omega)+\sigma^{2}}
$$

where $\beta(\omega)$ is an amplitude-attenuated operator and $\sigma^{2}$ is the regularization factor. In time-space domain, the regularization method can be expressed as

$$
\Lambda(\mathbf{k})=\frac{e^{-\alpha(\mathbf{k}) L_{F}}}{\left(e^{-\alpha(\mathbf{k}) L_{F}}\right)^{2}+\sigma^{2}}
$$

in forward wavefield propagation or

$$
\Lambda(\mathbf{k})=\frac{e^{-\alpha(\mathbf{k}) L_{B}}}{\left(e^{-\alpha(\mathbf{k}) L_{B}}\right)^{2}+\sigma^{2}},
$$

in backward wave propagation. From Eqs. (2.6)-(2.8), the regularization factor $\sigma^{2}$ is experiential, therefore the actual amplitude cannot be recovered accurately. From above, the crosscorrelation imaging condition with low-pass filtering or regularization methods are difficult to control the instability of Q-RTM. The right approaches should adopt the amplitude attenuated operator, not the amplitude amplified operator during the wavefield propagation of $Q-R T M$.

\subsection{Excitation amplitude imaging condition}

Considering the weakness of crosscorrelation imaging condition, in this paper, we propose a stable $Q-R T M$ algorithm based on the excitation amplitude imaging condition. Claerbout defined the reflective imaging condition for an impulsive wavelet as the dimensionless amplitude ratio [4]

$$
I(\mathbf{x})=\left.\frac{S(\mathbf{x}, t)}{R(\mathbf{x}, t)}\right|_{t=t_{i}}=\left.\frac{S(\mathbf{x}, t) R(\mathbf{x}, t)}{R(\mathbf{x}, t) R(\mathbf{x}, t)}\right|_{t=t_{i}},
$$

between the source wavefield $S(\mathbf{x}, t)$ and receiver wavefield $R(\mathbf{x}, t)$ at the imaging time $t_{i}=t_{i}(\mathbf{x}, t)$ corresponding to excitation time step array for the source. 
As shown in Figs. 1(a) and 1(b), the received seismic data in acoustic media and viscous media are expressed as $R(\mathbf{x}, t)$ and $R_{V}(\mathbf{x}, t)$, respectively, and $R_{V}(\mathbf{x}, t)$ can be expressed as

$$
R_{V}(\mathbf{x}, t)=R(\mathbf{x}, t) e^{-\alpha L_{F}} e^{-\alpha L_{B}} .
$$

In our stable $Q$-RTM algorithm, in order to recover the accurate reflection coefficient, we design three different kinds of forward and backward wavefields at the reflector. As shown in Fig. 1(d), we apply the attenuation factor of $e^{-\alpha L_{F}}$ to the source wavefield from the source to the reflector, and obtain the attenuated wavefield $S^{C}(\mathbf{x}, t)$ at the reflector as

$$
S^{C}(\mathbf{x}, t)=S(\mathbf{x}, t) e^{-\alpha L_{F}}
$$

For the backward wavefield extrapolation in Q-RTM along the path of $L_{B}$, we have two kinds of wavefields, one is the attenuated wavefield by applying the factor of $e^{-\alpha L_{B}}$, another is the non-attenuated wavefield. Thus, at the reflector the two wavefields from the receiver to the reflector, as illustrated in Fig. 1(d), can be written as

$$
R^{C_{1}}(\mathbf{x}, t)=R_{V}(\mathbf{x}, t) e^{-\alpha L_{B}}
$$

and

$$
R^{C_{2}}(\mathbf{x}, t)=R_{V}(\mathbf{x}, t)
$$

From Eqs. (2.11)-(2.13), we can obtain the up-going wavefields $R^{C_{1}}(\mathbf{x}, t), R^{C_{2}}(\mathbf{x}, t)$ and the down-going wavefield $S^{C}(\mathbf{x}, t)$ at the imaging time $t_{i}$. To recover the reflector coefficient, we use the wavefield $R^{C_{2}}(\mathbf{x}, t)$ to replace the down-going wavefield $D(\mathbf{x}, t)$ of the denominator in Eq. (2.9), and the $R^{C_{1}}(\mathbf{x}, t)$ and $S^{C}(\mathbf{x}, t)$ for the down-going wavefield $D(\mathbf{x}, t)$ in the numerator and the source wavefields, respectively. Then we obtain the compensated excitation amplitude imaging condition

$$
I^{C}(\mathbf{x})=\left.\frac{S^{C}(\mathbf{x}, t) R^{C_{1}}(\mathbf{x}, t)}{R^{C_{2}}(\mathbf{x}, t) R^{C_{2}}(\mathbf{x}, t)}\right|_{t=t_{i}},
$$

where $I^{C}(\mathbf{x})$ represents the compensated image. Substituting Eqs. (2.10)-(2.13) into Eq. (2.14), and after some algebraic manipulations, we obtain the compensated image as

$$
I^{C}(\mathbf{x})=\left.\frac{S(\mathbf{x}, t)}{R(\mathbf{x}, t)}\right|_{t=t_{i}} .
$$

It is seen from Eq. (2.15) that the compensated image $I^{C}(\mathbf{x})$ in $Q-R T M$ is theoretically equivalent to $I(\mathbf{x})$ of the acoustic RTM in Eq. (2.9). In other words, the reflector coefficient can be recovered. 


\subsection{Normalized crosscorrelation imaging condition}

The crosscorrelation image can be normalized by the square of the source or the receiver illumination strength $[4,12]$

$$
I(\mathbf{x})=\frac{\int_{0}^{T} S(\mathbf{x}, t) R(\mathbf{x}, t) d t}{\int_{0}^{T} R(\mathbf{x}, t) R(\mathbf{x}, t) d t} .
$$

Sun et al. adopted the following equation to compensate the $Q$ effects based on Eq. (2.16) [18]

$$
I^{C}(\mathbf{x})=\frac{\int_{0}^{T} S^{C}(\mathbf{x}, t) R^{C_{1}}(\mathbf{x}, t) d t}{\int_{0}^{T} R^{C_{2}}(\mathbf{x}, t) R^{C_{2}}(\mathbf{x}, t) d t} .
$$

Substituting Eqs. (2.11)-(2.13) into Eq. (2.17), we have

$$
I^{C}(\mathbf{x})=\frac{\int_{0}^{T} R_{V}(\mathbf{x}, t) e^{-\alpha L_{B}} S(\mathbf{x}, t) e^{-\alpha L_{F}} d t}{\int_{0}^{T} R_{V}(\mathbf{x}, t) R_{V}(\mathbf{x}, t) d t} .
$$

Inserting Eq. (2.10) into Eq. (2.18), after some algebraic manipulations, we obtain

$$
I^{C}(\mathbf{x})=\frac{\int_{0}^{T} R(\mathbf{x}, t) e^{-\alpha L_{F}} e^{-\alpha L_{B}} e^{-\alpha L_{B}} S(\mathbf{x}, t) e^{-\alpha L_{F}} d t}{\int_{0}^{T} R(\mathbf{x}, t) e^{-\alpha L_{F}} e^{-\alpha L_{B}} R(\mathbf{x}, t) e^{-\alpha L_{F}} e^{-\alpha L_{B}} d t} .
$$

In Eq. (2.19), there are two operators of $e^{-\alpha L_{F}}$ and $e^{-\alpha L_{B}}$ in both the numerator and denominator, but they are all in the integral. Thus, the attenuation operator in the numerator cannot be equals to the ones in the denominator. Based on the analysis in this section, the normalized crosscorrelation imaging condition cannot obtain satisfied Q-RTM images. We will verify it in the subsection of "Graben model".

\subsection{Calculation of wavefields in viscoacoustic media}

In this subsection we discuss the calculation of the wavefields used in Eqs. (2.14) and (2.17).

Zhu et al. derived a viscoacoustic wave equation [31]. After that, Li et al. improved the applicability for small $Q$ values, and gave the following wave equation [14]

$$
\begin{aligned}
& \frac{1}{c^{2}} \frac{\partial^{2} p}{\partial t^{2}}+\theta_{1} \omega_{0}^{-2 \gamma}\left[c_{0}^{2 \gamma} \phi\left(-\nabla^{2}\right)^{\gamma+1}+c_{0}^{2 \gamma-1} \delta \frac{\partial}{\partial t}\left(-\nabla^{2}\right)^{\gamma+1 / 2}\right] p \\
& +\theta_{2} \omega_{0}^{-2 \gamma}\left[c_{0}^{2 \gamma} \alpha\left(-\nabla^{2}\right)^{\gamma+1}+c_{0}^{2 \gamma-1} \beta \frac{\partial}{\partial t}\left(-\nabla^{2}\right)^{\gamma+1 / 2}-\nabla^{2}\right] p-\nabla^{2} p=0
\end{aligned}
$$


where

$$
\begin{aligned}
& \alpha=\cos ^{2 \gamma+2} W[\sin (\pi \gamma) \sin V \cos W+\cos (\pi \gamma) \cos U], \\
& \beta=\cos ^{2 \gamma} W[\sin (\pi \gamma) \cos V \cos W-\cos (\pi \gamma) \sin U], \\
& \phi=\cos (\pi \gamma)-\cos ^{2 \gamma+2} W[\sin (\pi \gamma) \sin V \cos W+\cos (\pi \gamma) \cos U], \\
& \delta=\sin (\pi \gamma)-\cos ^{2 \gamma} W[\sin (\pi \gamma) \cos V \cos W-\cos (\pi \gamma) \sin U],
\end{aligned}
$$

and $U=\pi \gamma(\gamma+1), V=\pi \gamma(2 \gamma+1) / 2, W=\pi \gamma / 2, p$ is the wavefield, $x$ and $z$ are space coordinate, $\theta_{1}$ and $\theta_{2}$ are constants, $\nabla^{2}=\partial^{2} / \partial x^{2}+\partial^{2} / \partial z^{2}, \gamma=1 / \pi Q$, and $0<\gamma(\mathbf{x})<0.5$ in real media, $c^{2}=M_{0} / \rho=c_{0}^{2} \cos ^{2}(\pi \gamma / 2), c_{0}$ and $\rho$ are the reference velocity and density, $\omega_{0}$ is the reference angular frequency, $M_{0}$ represents the bulk modulus. When $\theta_{1}=-1$ and $\theta_{2}=-1$, Eq. (2.20) becomes

$$
\begin{aligned}
& \frac{1}{c^{2}} \frac{\partial^{2} p}{\partial t^{2}}-\omega_{0}^{-2 \gamma}\left[c_{0}^{2 \gamma} \phi\left(-\nabla^{2}\right)^{\gamma+1}+c_{0}^{2 \gamma-1} \delta \frac{\partial}{\partial t}\left(-\nabla^{2}\right)^{\gamma+1 / 2}\right] p \\
& -\omega_{0}^{-2 \gamma}\left[c_{0}^{2 \gamma} \alpha\left(-\nabla^{2}\right)^{\gamma+1}+c_{0}^{2 \gamma-1} \beta \frac{\partial}{\partial t}\left(-\nabla^{2}\right)^{\gamma+1 / 2}-\nabla^{2}\right] p-\nabla^{2} p=0,
\end{aligned}
$$

which stands for the viscoacoustic wave equation. Then, Eq. (2.20) can be written as

$$
\frac{1}{c^{2}} \frac{\partial^{2} p_{A}}{\partial t^{2}}-\omega_{0}^{-2 \gamma} c_{0}^{2 \gamma} \phi\left(-\nabla^{2}\right)^{\gamma+1} p_{A}-\omega_{0}^{-2 \gamma} c_{0}^{2 \gamma-1} \delta \frac{\partial}{\partial t}\left(-\nabla^{2}\right)^{\gamma+1 / 2} p_{A}-\nabla^{2} p_{A}=0,
$$

which describes only the amplitude attenuation during the wave propagation, where $P_{A}$ stands for the amplitude attenuation only wavefield. And when $\theta_{1}=0$ and $\theta_{2}=-1$, Eq. (2.20) can be written as

$$
\frac{1}{c^{2}} \frac{\partial^{2} p_{p}}{\partial t^{2}}-\omega_{0}^{-2 \gamma} c_{0}^{2 \gamma} \alpha\left(-\nabla^{2}\right)^{\gamma+1} p_{p}-\omega_{0}^{-2 \gamma} c_{0}^{2 \gamma-1} \beta \frac{\partial}{\partial t}\left(-\nabla^{2}\right)^{\gamma+1 / 2} p_{p}=0,
$$

which represents the viscoacoustic wave equation only with the phase dispersion, $p_{p}$ represents the phase dispersion only wavefield in simulation.

\subsection{Implementation of stable $Q$-RTM}

The complete implementation of our stable Q-RTM algorithm consists of three steps.

Step 1: Forward extrapolating the source wavefield. We solve Eq. (2.22) to propagate the source wavefield with a given source wavelet. In this step, we can calculate the imaging time $t_{i}$ according to the maximum amplitude of the wavefields, and obtain the corresponding attenuated wavefield $S^{C}\left(\mathbf{x}, t_{i}\right)$, the imaging time and its corresponding amplitude are saved in the memory.

Step 2: Backward extrapolating the receiver wavefield. By solving Eqs. (2.22) and (2.24), we back-propagate the receiver data $R_{V}\left(\mathbf{x}, t_{i}\right)$ to the imaging time, which has been saved 
in the memory in Step1. Then, we can obtain the wavefield $R^{C_{1}}\left(\mathbf{x}, t_{i}\right)$ and $R^{C_{2}}\left(\mathbf{x}, t_{i}\right)$ by solving Eq. (2.22) and (2.24), respectively.

Step 3: Applying the excitation amplitude imaging condition. Based on the wavefields which we have obtained in the above two steps, the imaging condition of Eq. (2.14) is applied to get a stable $Q$-RTM result.

\section{Migration examples}

In this section, we try to use a simple graben model, realistic BP gas model and a field seismic data to illustrate the accuracy, stability and anti-noise capability of our stable $Q-$ RTM algorithm. The source is a Ricker wavelet with a dominant frequency of $20 \mathrm{~Hz}$, and the reference frequency is $200 \pi$ times of the dominant frequency for all the migration examples. The acoustic RTM profiles obtained using synthetic data without absorption are regarded as references images.

\subsection{Graben model}

Fig. 2 shows the graben model, which is discretized into $256 \times 256$ grids with the grid size of $10 \mathrm{~m} \times 10 \mathrm{~m}$, the velocity and $Q$ of each layer are given in this figure. There are 81 sources, which are located at a depth of $20 \mathrm{~m}$ and uniformly set in horizontal direction with an interval of $30 \mathrm{~m}$. The temporal sampling interval is $1 \mathrm{~ms}$ and the recording length is $2.5 \mathrm{~s}$.

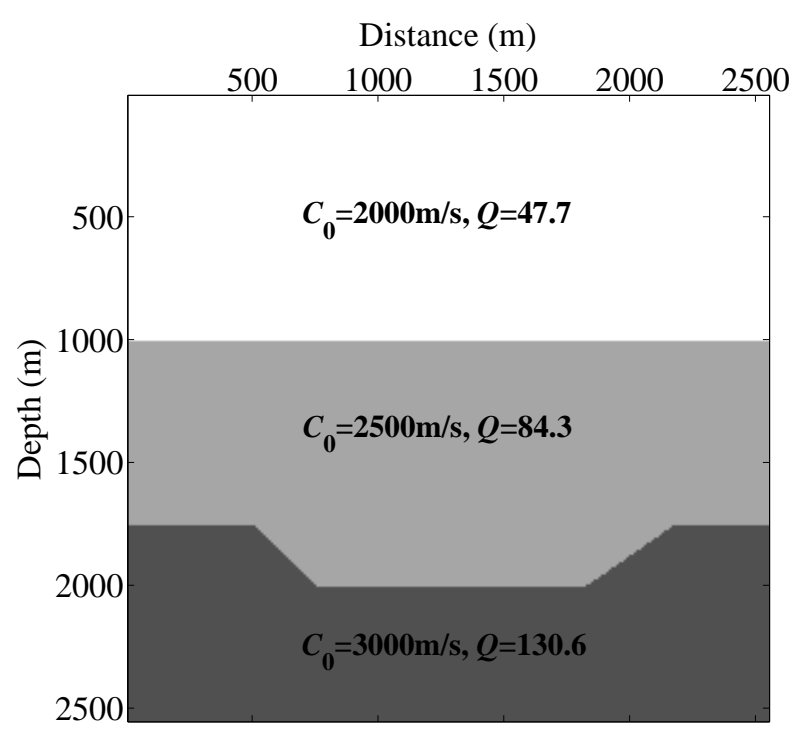

Figure 2: A graben velocity and $Q$ model. 


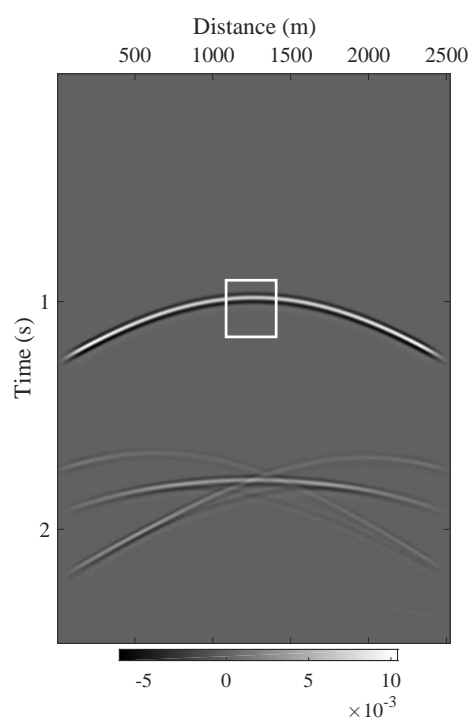

(a)

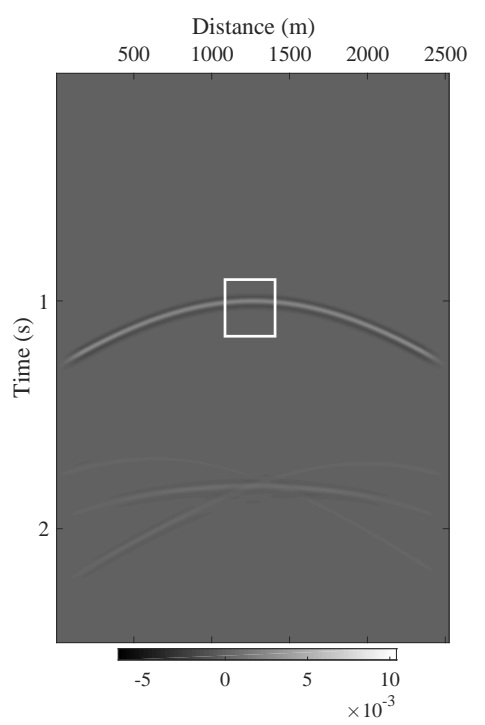

(b)

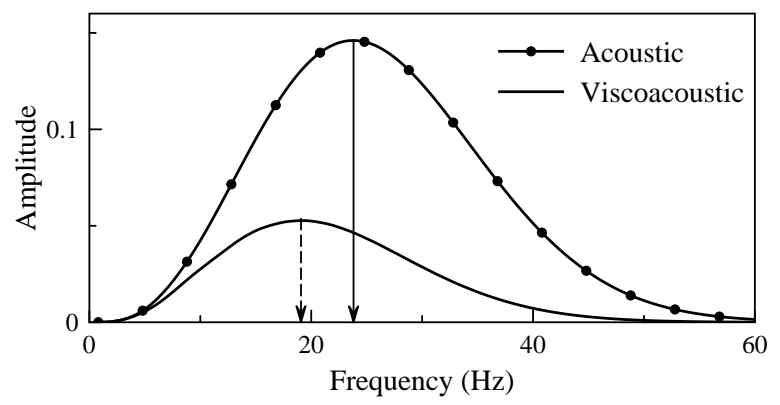

(c)

Figure 3: Two direct wave muted shot gathers of the graben model for (a) acoustic case, and (b) viscoacoustic case. (c) The averaged amplitude spectra of the signal in the white boxes in (a) and (b), the solid line and solid line with dots stand for the spectrum of viscoacoustic and acoustic simulation results, respectively.

Figs. 3(a) and 3(b) show two direct wave muted acoustic and viscoacoustic synthetic shot gathers, respectively. The source is located at the position of $(1200 \mathrm{~m}, 20 \mathrm{~m})$. Due to the viscous effect, the energy of the wavefield shown in Fig. 3(b) is attenuated, and the time from the source to the interface is extended because of the phase dispersion. Fig. 3(c) displays the amplitude spectra of the data in the white boxes of Figs. 3(a) and 3(b), from which we can clearly see the energy attenuation and the decrease of the dominant frequency. The attenuation is stronger at high frequencies than at low frequencies. The stronger loss at high frequencies will cause low resolution of RTM images.

Fig. 4 shows the time map and excitation amplitude for a source at $(1200 \mathrm{~m}, 20 \mathrm{~m})$. The imaging time $t_{i}$ is the arrival time of the maximum amplitude. In the backward wave propagation of $Q$-RTM, we can obtain the attenuated receiver wavefields $R^{C_{1}}\left(\mathbf{x}, t_{i}\right)$ and 


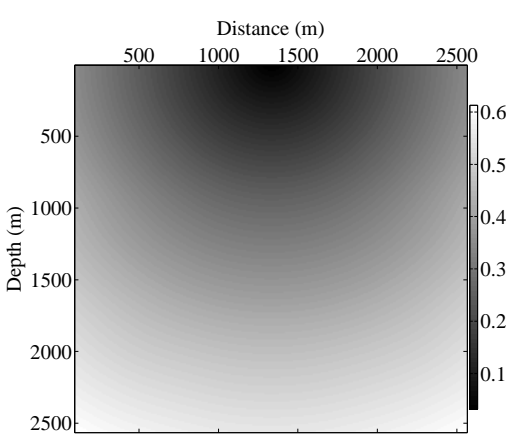

(a)

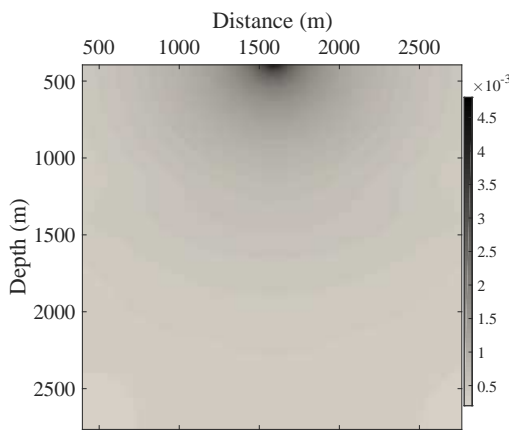

(b)

Figure 4: Excitation (a) time and (b) amplitude maps for a single source located at the center of Fig. 2. The amplitude in (b) has been clipped to reduce the effect of the plotting scale near the source point.

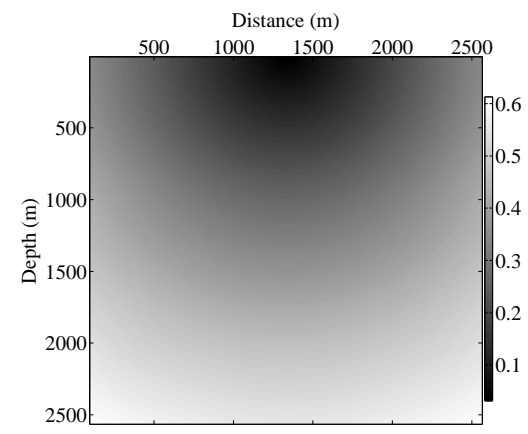

(a)

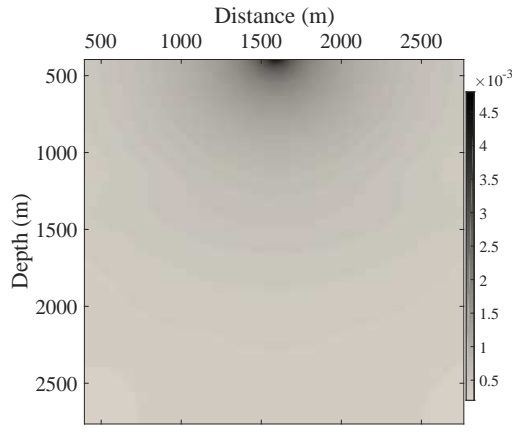

(b)

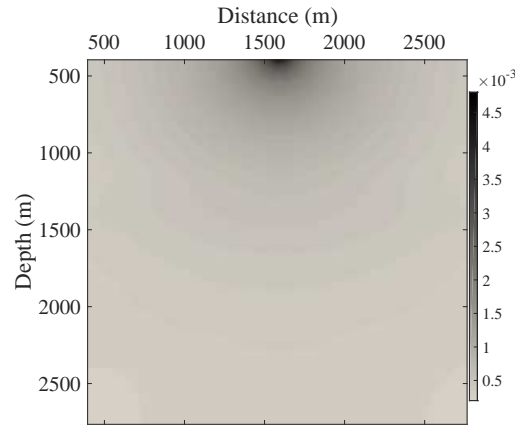

(c)

Figure 5: (a) The acoustic RTM image with acoustic seismic data, (b) the acoustic RTM image of viscoacoustic seismic data, and (c) our stable $Q-$ RTM image of viscoacoustic seismic data.

$R^{C_{2}}\left(\mathbf{x}, t_{i}\right)$. Using the excitation amplitude imaging condition, we will recover the reflector coefficient of the model.

Fig. 5(a) shows the reference migration image from acoustic seismic data, Fig. 5(b) shows the non-compensated RTM image from viscoacoustic seismic data, and Fig. 5(c) 


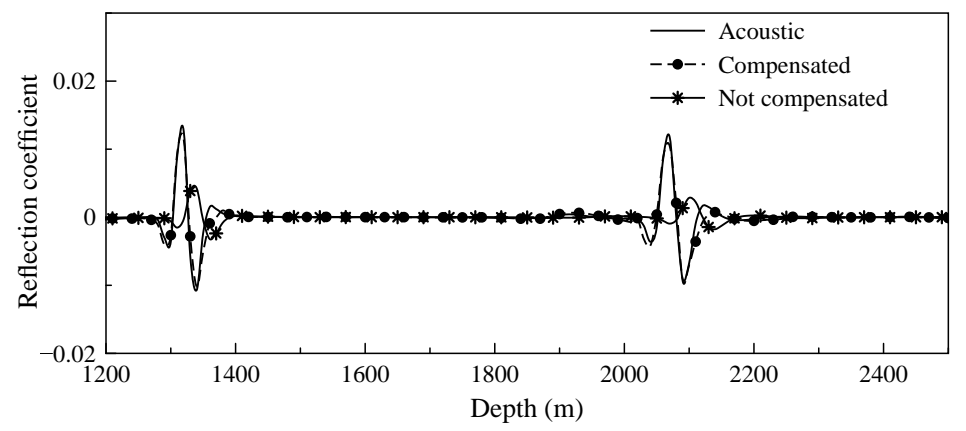

(a)

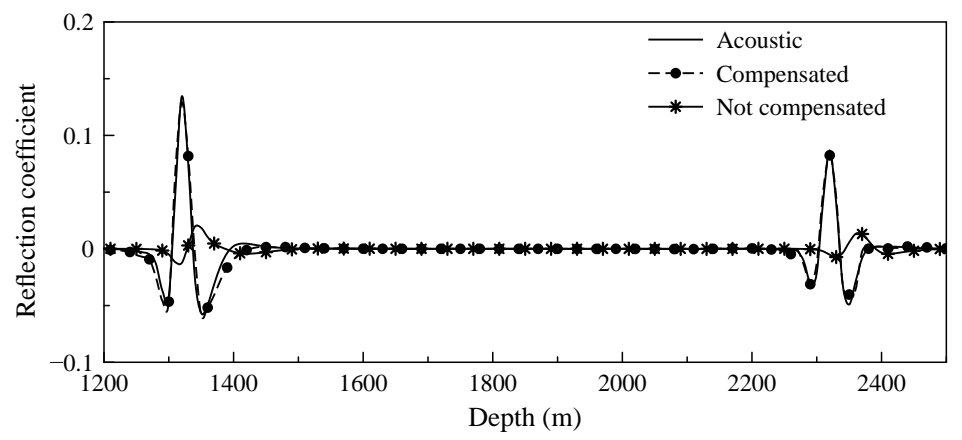

(b)

Figure 6: Traces extracted from Fig. 5 at the position of (a) $x=100 \mathrm{~m}$ and (b) $x=900 \mathrm{~m}$. The solid line and dashed line with dots stand for the reference and compensated images, respectively. The solid line with stars shows the non-compensated image.

depicts the absorption compensated migration image by our stable Q-RTM algorithm. It is noticed from Fig. 5(b) that the deeper interface cannot be well imaged because of the amplitude attenuation. After our stable $Q$-RTM processing, the reflector coefficient can be recovered as shown in Fig. 5(c) compared to Fig. 5(a), and the second interface is recovered. Moreover, our stable Q-RTM image matches the reference one very well.

To observe the compensated effects clearly, we extract two traces from Figs. 5(a), 5(b) and 5(c) at $x=100 \mathrm{~m}$ and $900 \mathrm{~m}$, and show them in Figs. 6(a) and 6(b), respectively. It is seen from Fig. 6 that the waveform of the reference image (the solid line) is close to that of compensated one (the dashed line with dots). The solid line with stars (non-compensated image) illustrates that we obtain inaccurate stratum position and reflection coefficient. Both Figs. 5 and 6 demonstrate that our stable $Q$-RTM method is quite accurate.

Next, we test the normalized crosscorrelation imaging condition given in Eq. (2.19). Figs. 7(a) and 7(b) show the reference and compensated images with crosscorrelation imaging condition of Eqs. (2.16) and (2.19), respectively. It is seen from Figs. 7(a) and 7(b) that the energy of the deeper layer in Fig. 7(b) is weaker than the reference one in Fig. 7(a). To see the compensation effect more clearly, we extract a trace waveform at $x=900 \mathrm{~m}$ from Figs. 7(a) and 7(b), and show them in Fig. 7(c), where the solid line and dashed line 


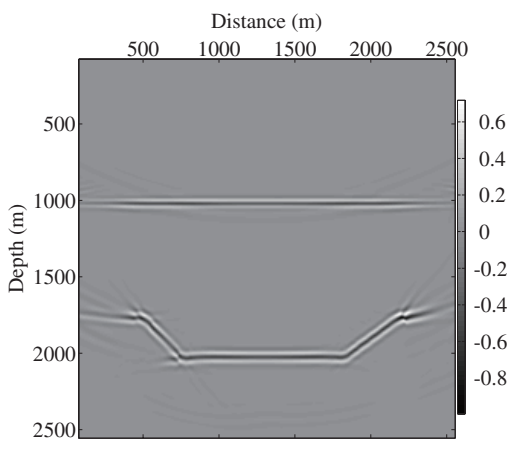

(a)

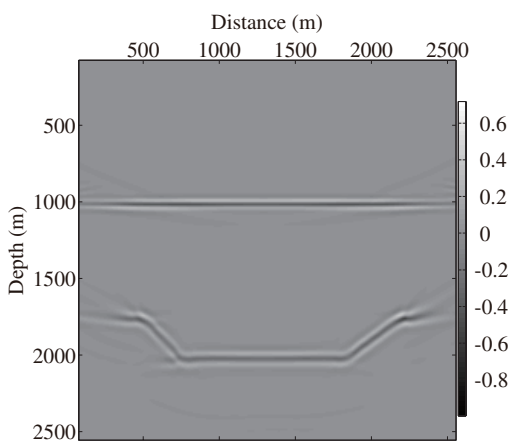

(b)

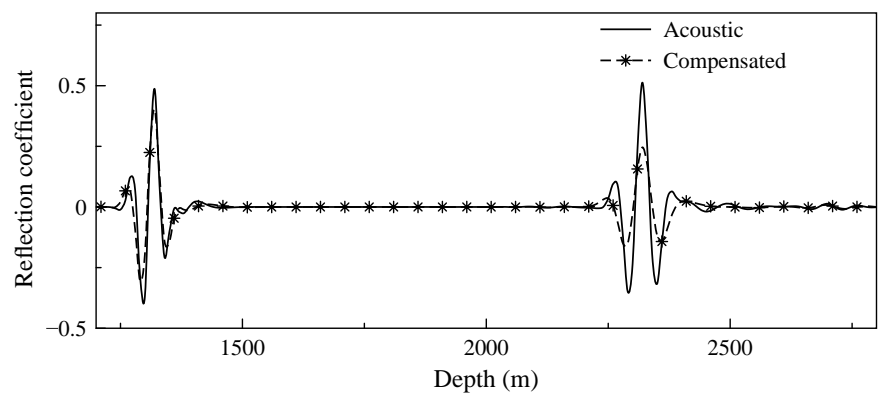

(c)

Figure 7: (a) The acoustic RTM image with acoustic seismic data using the imaging condition of Eq. (2.21), (b) the compensated image of viscoacoustic seismic data using the imaging condition of Eq. (2.23), and (c) a trace extracted from (a) and (b) at $x=900 \mathrm{~m}$. The solid line and dashed line with stars represent the reference and compensated images with the normalized crosscorrelation imaging condition, respectively.

with stars stand for the reference and compensated images, respectively. We can see that the normalized crosscorrelation imaging condition can compensate the phase dispersion, the actual position of the layers can be obtained, there are still large gap between the two lines, and the amplitude attenuation cannot be compensated well. Thus, the normalized crosscorrelation imaging condition is not suitable for $Q$-RTM algorithm.

\subsection{BP gas model}

To further demonstrate the stability, accuracy and noise immunity of our proposed QRTM method, we test our method on the BP gas chimney model with the clean data and noisy data, respectively. Figs. 8(a) and 8(b) show its velocity and $Q$ models [30], which contains a high attenuation zone caused by a gas chimney with $Q=20$, which is shown in the black box in Fig. 8(b). The model has $398 \times 161$ grids with a spatial interval of $d x=d z=10 \mathrm{~m}$. The time sampling interval is $1 \mathrm{~ms}$. In the observation system, the 80 sources with an interval of $40 \mathrm{~m}$ from the left to the right are distributed laterally, all the sources and receivers are located at a depth of $30 \mathrm{~m}$, the records lasts 2.0s. 


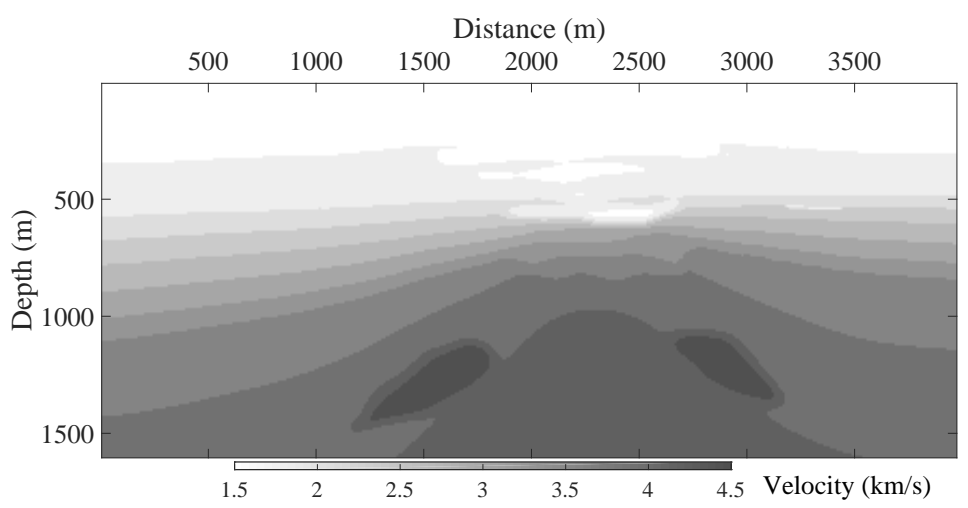

(a)

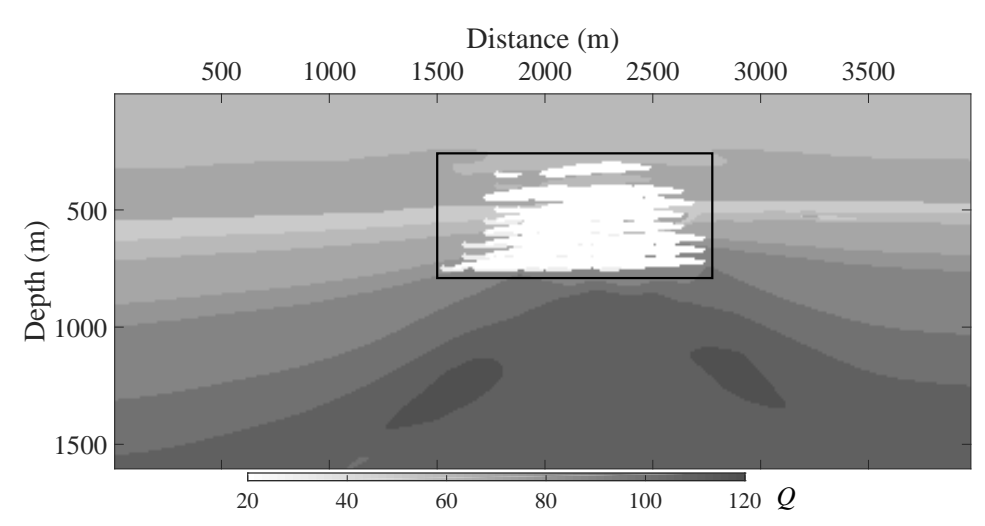

(b)

Figure 8: The BP gas model of (a) velocity and (b) $Q$.

\section{$Q$-RTM with clean data}

In this example, we first consider our $Q$-RTM for BP gas chimney model without any noise. Figs. 9(a) and 9(b) shows the synthetic shot gathers in an acoustic medium and in a viscoacoustic medium without any noises respectively, we can observe apparent amplitude decay in viscoacoustic gather because of the gas chimney attenuation.

We perform three kinds of RTMs. Figs. 10(a) and 10(b) show the acoustic RTM with acoustic and viscoacoustic seismic data, respectively, and Fig. 10(c) illustrates the stable Q-RMT image with viscoacoustic data. As a reference image shown in Fig. 10(a), the anticline structure below the high-attenuation gas chimney can be clearly seen. Compared Fig. 10(b) to Fig. 10(a), the interfaces in the white box are punch-drunk and hard to be recognized because of the amplitude attenuation and phase dispersion. However, for our stable Q-RTM image of Fig. 10(c), the energy of the reflections, e.g., those in the white box looks the same as the reference one (Fig. 10(a)). From this example we also know that our stable $Q$-RTM method can obtain satisfactory compensated images for strong absorption media. 


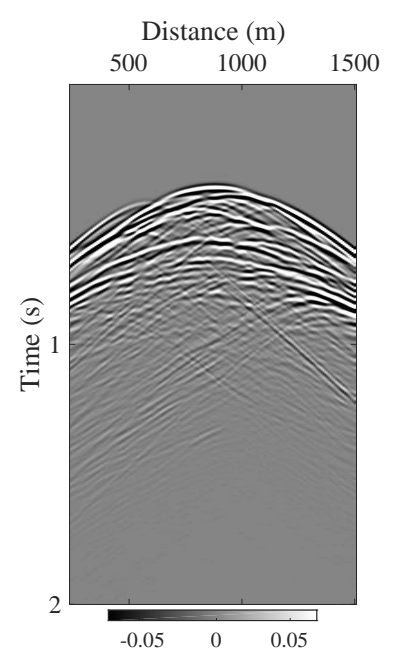

(a)

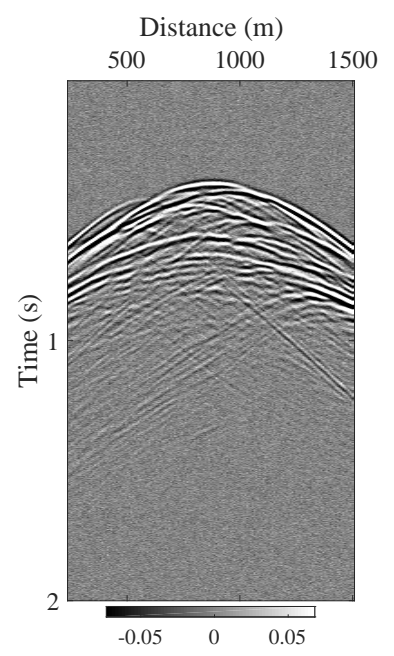

(c)

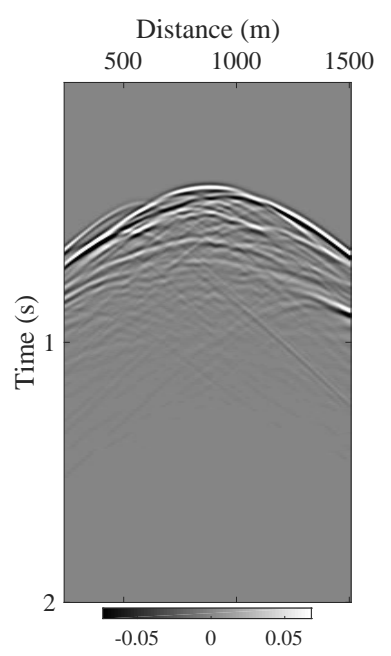

(b)

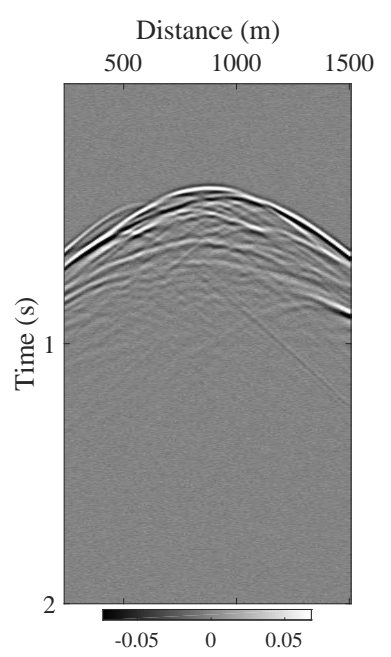

(d)

Figure 9: Shot gathers of BP gas model for (a) acoustic, (b) viscoacoustic cases without noises, (c) the acoustic and $(d)$ viscoacoustic cases with $20 \mathrm{~dB}$ random noises.

It should be noted that in the existing Q-RTMs, the low-pass filter is usually utilized to suppress the amplification of noise during attenuation compensation. The selection of its cutoff frequency is based on the noise level in the power spectrum of the measured data, and is hard to conduct. Our Q-RTM algorithm is relative stable, without taking any other measures, such as low-pass filtering or regularization.

Fig. 11 shows the traces extracted from Fig. 10 at $x=900 \mathrm{~m}$ and $x=1900 \mathrm{~m}$. The solid line is from Fig. 10(a), the lines with dots and stars are from Figs. 10(c) and 10(b), respec- 


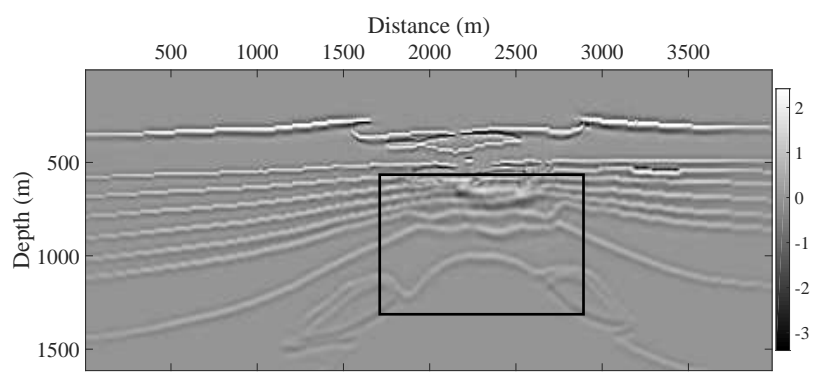

(a)

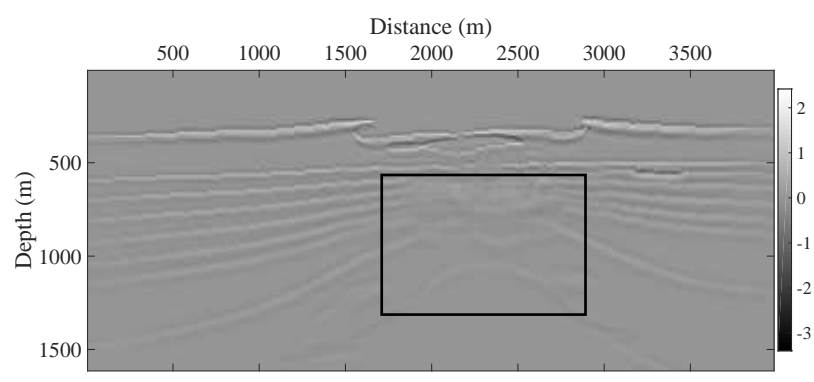

(b)

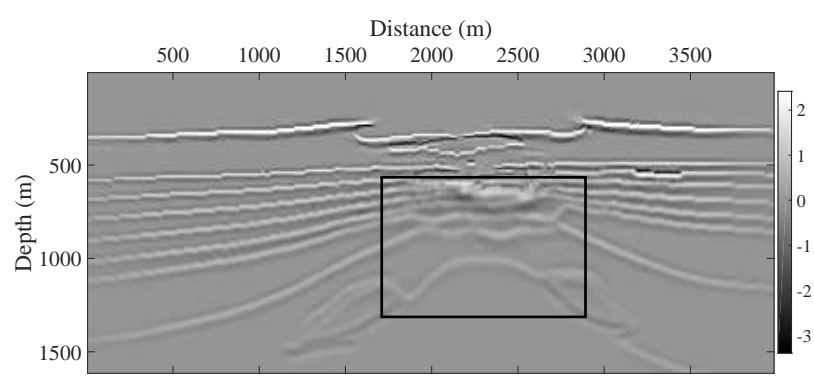

(c)

Figure 10: (a) Acoustic RTM reference image with acoustic clean data, (b) acoustic RTM image with viscoacoustic clean data, and (c) the stable Q-RTM image with viscoacoustic clean data.

tively. We can see that the compensated lines match the reference lines very well. It means that the amplitude attenuation and phase dispersion are recovered successfully. But the non-compensated line does not match the reference one, in both the energy and waveform position. In Fig. 11(a), there are two dashed arrows and two solid arrows, which represent the same event of the compensated and non-compensated RTM images. The distance between the dashed arrows is $72.7 \mathrm{~m}$, and that between the solid arrows is 90.9 $\mathrm{m}$. The gap of the compensated events is narrower than that of the non-compensated one. 


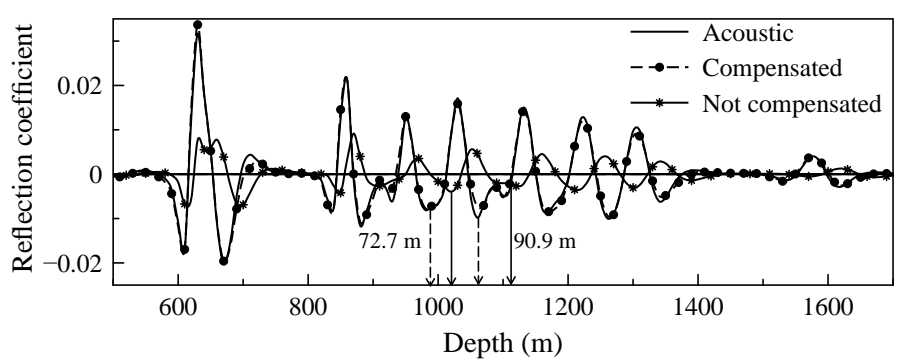

(a)

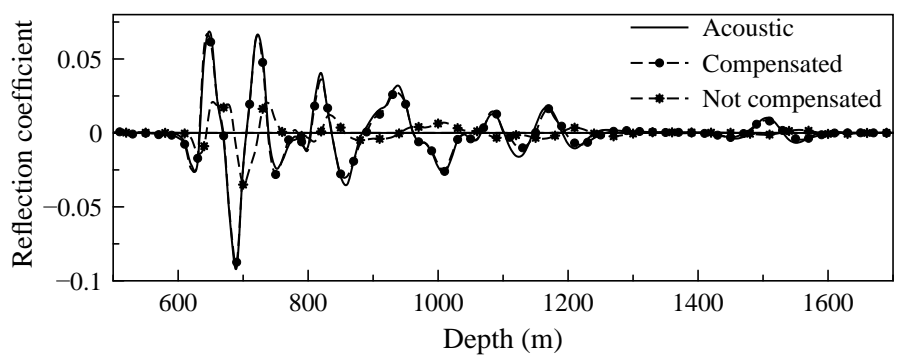

(b)

Figure 11: The waveform plot of traces located at (a) $x=900 \mathrm{~m}$ and (b) $x=1900 \mathrm{~m}$ from Fig. 10. The solid line is from the acoustic RTM image with acoustic data, the lines with dots and stars stand for the traces of stable $Q-R T M$ and non-compensated RTM, respectively.

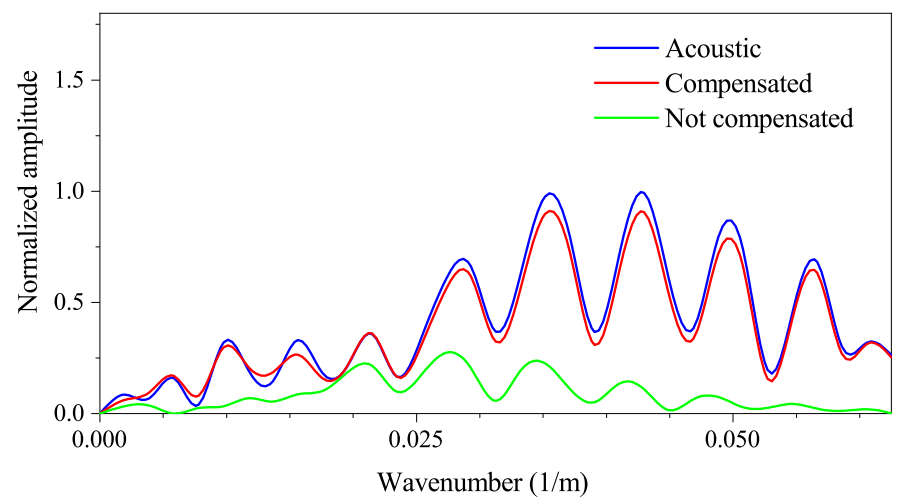

Figure 12: The wavenumber spectra of different migration images within the white boxes shown in Fig. 10. The blue and red lines represent the spectra of reference and stable $Q-R T M$ images, respectively. The green line stands for the spectrum of the non-compensated image.

From Fig. 12, which is the wavenumber spectra of different migration images, we can also see that the frequency band is expanded, and the dominant frequency is increased for the compensated case. Thus, our stable $Q$-RTM method can improve the resolution of the migration images compared to the non-compensated case. 


\section{$Q-R T M$ with noise data}

In order to facilitate the comparison between the crosscorrelation imaging condition with low-pass filtering and our proposed stable $Q$-RTM method in anti-noise performance and resolution enhancement, we further consider $Q$-RTM for the noisy data, we add $20 \mathrm{~dB}$ band-pass filtered random noise to the seismic records. Figs. 9(c) and 9(d) show the shot gathers in acoustic medium and viscoacoustic medium with $20 \mathrm{~dB}$ noises, respectively.

Fig. 13(a) shows the reference migration image with the noisy acoustic seismic data (Fig. 9(c)), and Figs. 13(b) and 13(c) demonstrate the non-compensated and compensated migration images obtained by our stable $Q$-RTM method, respectively. From which, we can see that, since we adopt the attenuated wavefields in both forward and backward

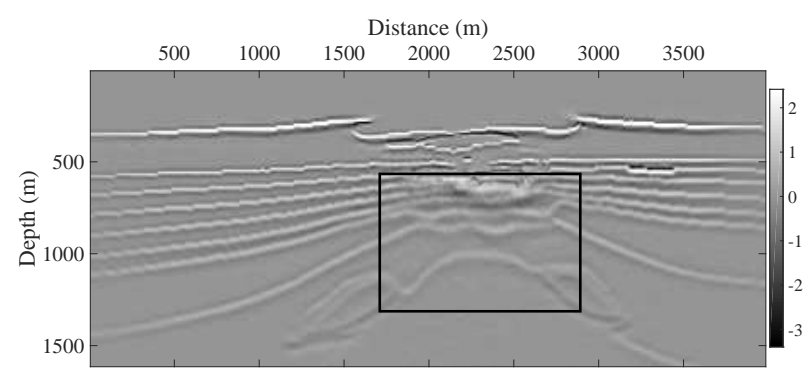

(a)

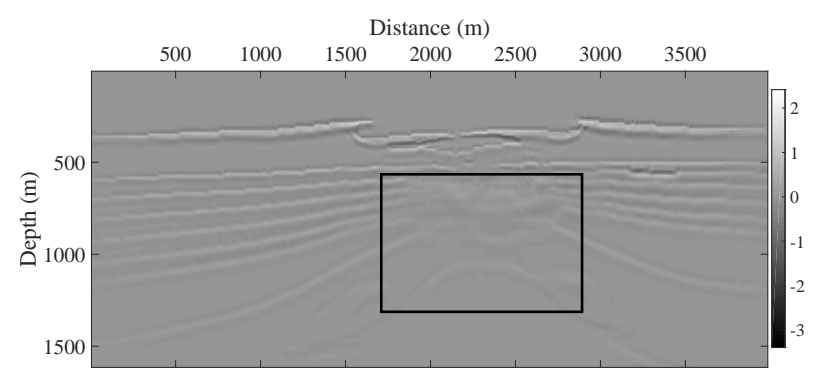

(b)

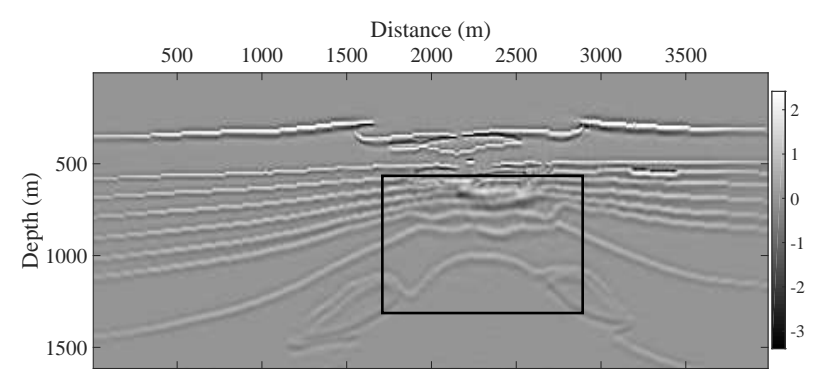

(c)

Figure 13: (a) Acoustic RTM reference image with acoustic noisy data, (b) acoustic RTM image with viscoacoustic noisy data, and (c) the stable Q-RTM image with viscoacoustic noisy data. 


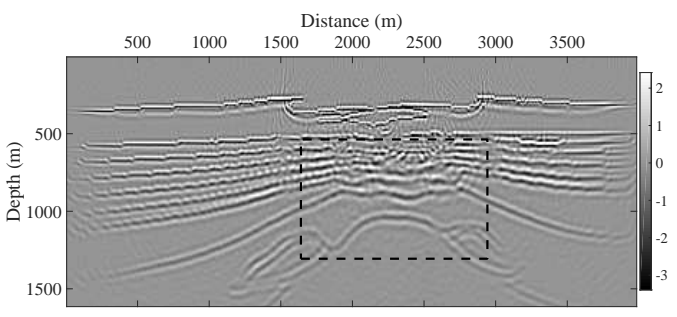

(a)

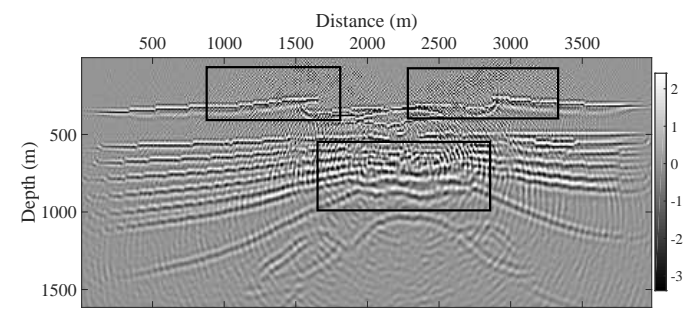

(b)

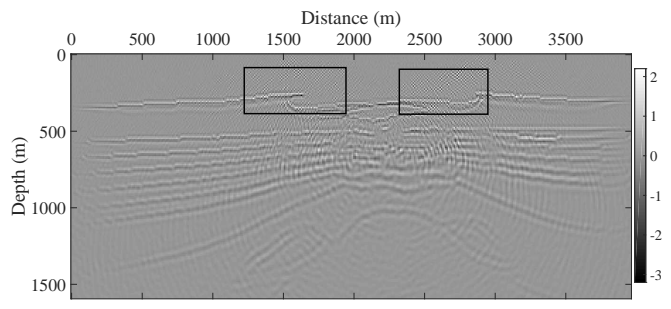

(c)

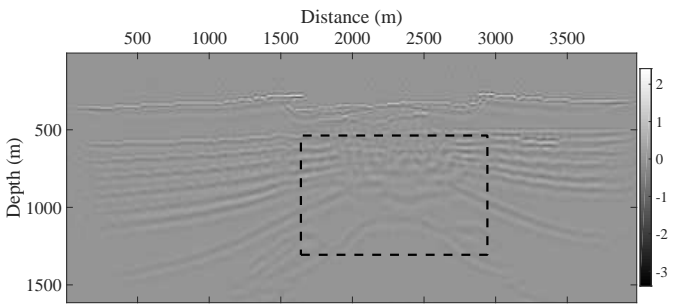

(d)

Figure 14: The crosscorrelation imaging condition with the amplitude amplified for (a) the reference RTM image, and the Q-RTM images with the cutoff frequency (b) $150 \mathrm{~Hz}$, (c) $100 \mathrm{~Hz}$ and (d) $50 \mathrm{~Hz}$, respectively.

wave propagation, our method can reach a perfect stabilization scheme throughout the whole $Q$-RTM process. Compare the compensated image (Fig. 13(c)) to the reference image (Fig. 13(a)), the two images matches well even with $20 \mathrm{~dB}$ noises. Thus, our stable $Q$-RTM is not only accurate but also has a strong noise resistance.

Fig. 14 demonstrate the RTM images with crosscorrelation imaging condition, which was shown in section "crosscorrelation imaging condition". The stability is controlled 
by low-pass filtering method, and the cutoff frequency is determined by the maximum velocity of the model. Fig. 14(a) displays the reference image with noise seismic data, and Figs. 14(b)-14(d) show the $Q$-RTM images with the crosscorrelation imaging condition whose amplitudes are exponential amplified, and the cutoff frequency are $150 \mathrm{~Hz}, 100 \mathrm{~Hz}$ and $50 \mathrm{~Hz}$, respectively. From Figs. 14(b) and 14(c), we can notice the strong instability in the solid rectangular boxes, which seriously affect the imaging quality. In order to suppress the instability, we must adopt the small enough cutoff frequency. In this model, for the sake of stability, the cutoff frequency should be less than $50 \mathrm{~Hz}$, which is shown in Fig. 14(d). Compare Fig. 14(d) to Fig. 14(a), we can see the great distance between the two images from the dotted rectangular boxes because of the low-pass filtering method. The crosscorrelation imaging condition with the amplitude amplified cannot balance the accuracy and anti-noise. Thus, this compensation method is unreliable.

\subsection{Field data example}

Here, we use a field data set to evaluate our stable $Q$-RTM method. The width and depth of the area are $15.61 \mathrm{~km}$ and $8.01 \mathrm{~km}$, respectively. There are 81 shots, unevenly distributed on the surface. Fig. 15 shows two $6 \mathrm{~s}$ long shot gathers located at $x=1300 \mathrm{~m}$ and $x=2300 \mathrm{~m}$. We divide the computation domain into $1561 \times 801$ grids. The spatial sampling interval is $10 \mathrm{~m}$. the time step is $0.5 \mathrm{~ms}$. A ricker wavelet with a center frequency of $20 \mathrm{~Hz}$ is used as an explosive source. The velocity and $Q$ models are shown in Fig. 16,

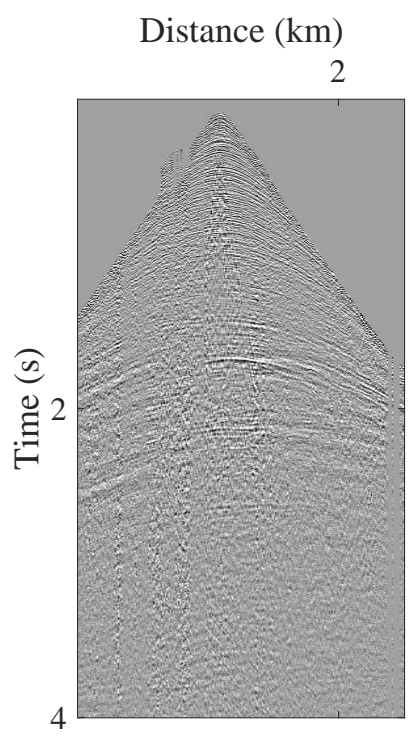

(a)

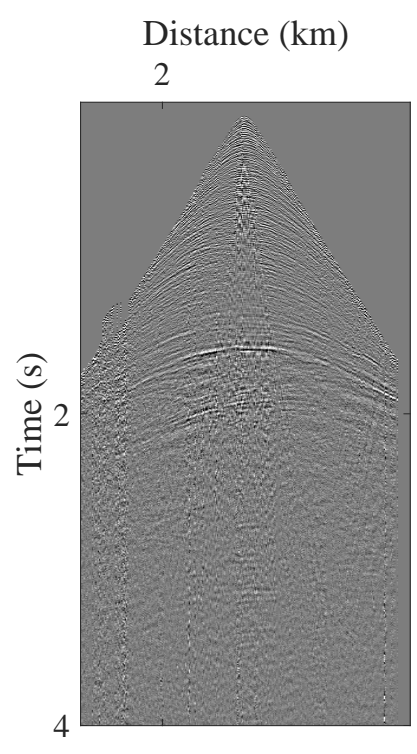

(b) Figure 15: Two shot gathers of field seismic data, the sources are located at (a) $x=1300 \mathrm{~m}$ and (b) $x=1300$
$\mathrm{~m}$. 


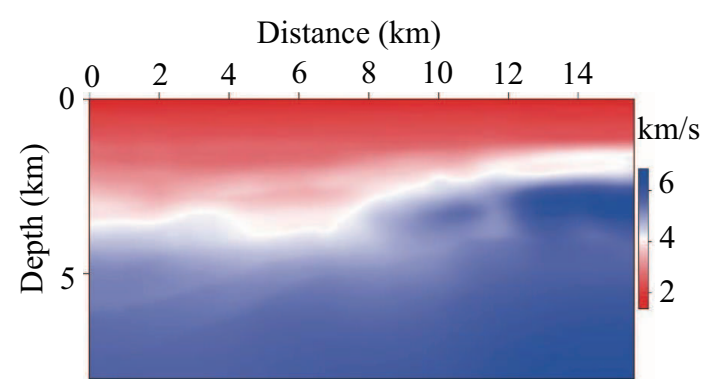

(a)

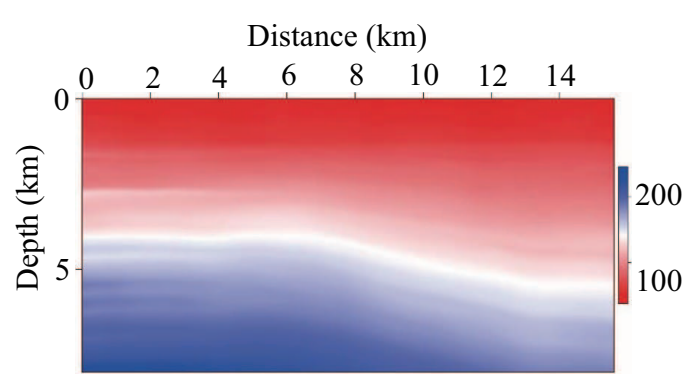

(b)

Figure 16: (a) Velocity and (b) $Q$ model of the field seismic data.

in which the value of the velocity range from $1500 \mathrm{~m} / \mathrm{s}$ to $6800 \mathrm{~m} / \mathrm{s}$, and that of $Q$ ranges from 17.5 to 283.6 .

Figs. 17(a) and 17(b) illustrate the conventional RTM and Q-RTM images, respectively. The cutoff frequency of Fig. 17(b) is $100 \mathrm{~Hz}$. Comparing Figs. 17(b) to 17(a), we can observe that after compensation with our method, the events are more continuous, and the identifiable horizons are richer, especially in area A and B. To see clearly, the enlarged images of area B are shown in Fig. 18, which also verify these for clear observations. From the wavenumber spectra (Fig. 19) of a trace extracted at $x=11.0 \mathrm{~km}$ of the migration images, we know that the frequency band is widened after compensation. It can explain the improvement of the resolution of migration image shown in Fig. 17(b).

\section{Discussion}

The stable $Q$-RTM algorithm is based on the excitation amplitude imaging condition. The amplitudes of both the forward and backward extrapolated wavefields are all attenuated, which is different from conventional Q-RTM schemes. As a result, our Q-RTM method is relative stable. In our algorithm, the forward wavefield at any time is not necessary to be saved into the hard disks, therefore the method has a high computation efficiency. In this paper, we use the maximum amplitude to calculate the excitation amplitude imaging 


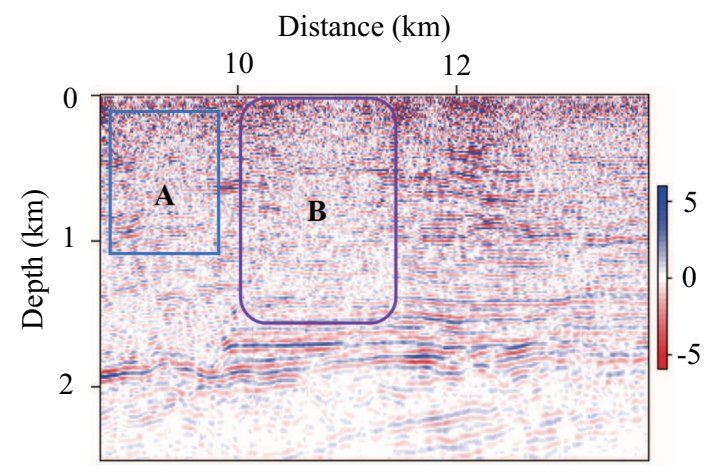

(a)

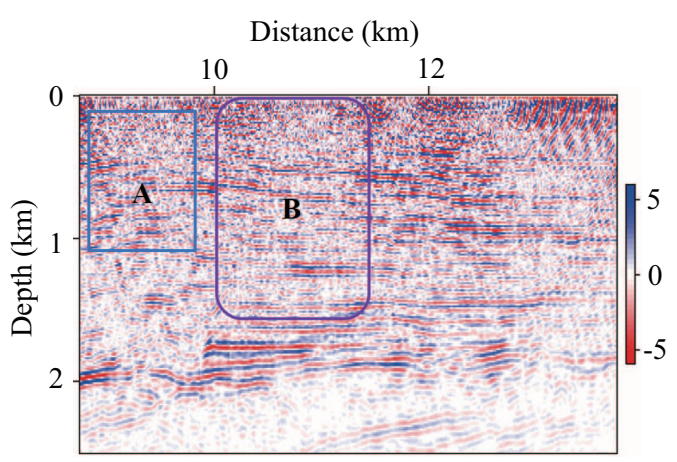

(b)

Figure 17: Migration images obtained by (a) acoustic RTM and (b) Q-RTM.

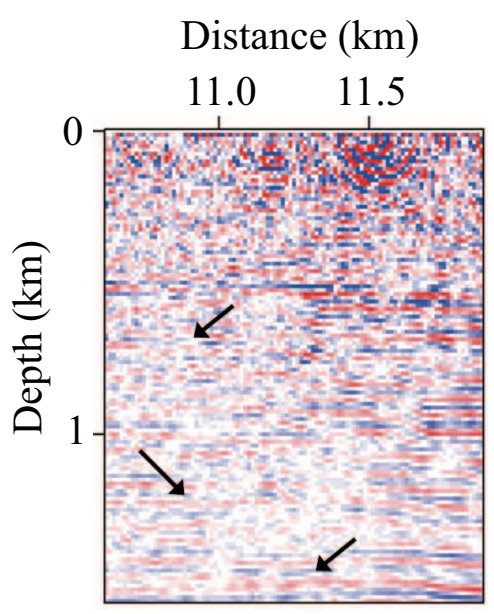

(a)

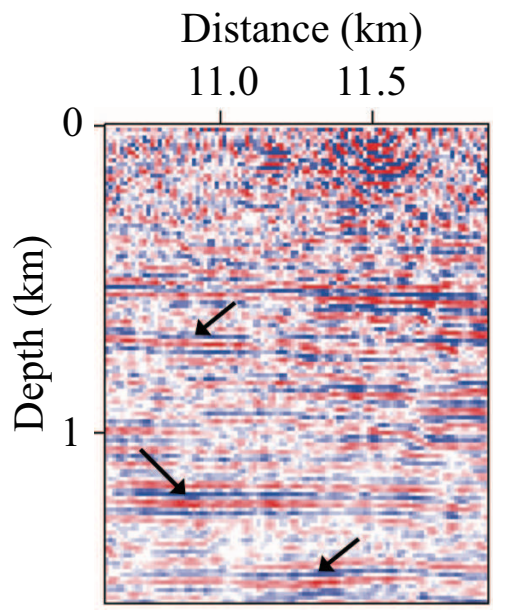

(b)

Figure 18: Comparisons of enlarged image of area B in Figs. 17(a) and 17(b). (a) acoustic RTM and (b) Our proposed Q-RTM. 


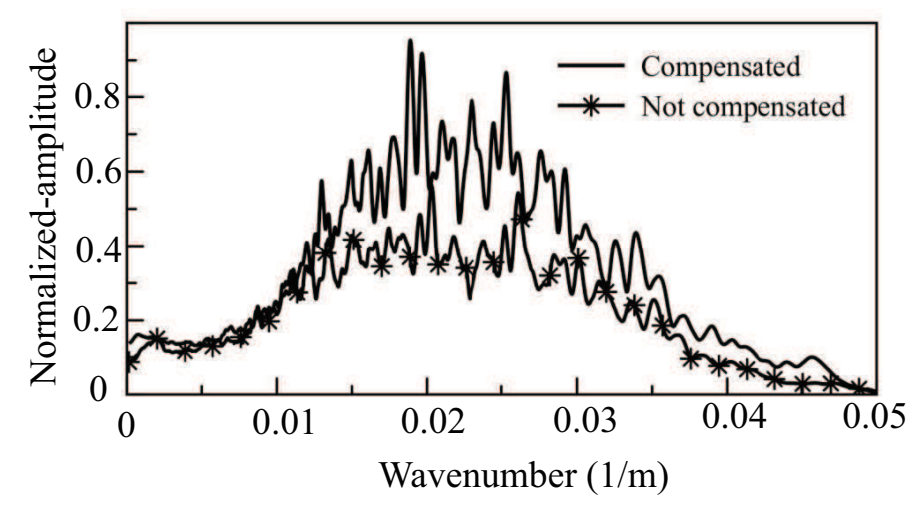

Figure 19: The wavenumber spectra of a trace extracted at $x=11 \mathrm{~km}$ from the images in Figs. $17(\mathrm{a})$ and 17 (b). The solid and solid lines with stars stand for those of compensated and not compensated images, respectively.

time, which may be not suitable for the long offset recording now. And the denominator of the excitation amplitude imaging condition may be zero, which causes the mutation in some imaging points. In our stable $Q-R T M$, we need to solve three wave equations, the computation amount will be larger than traditional Q-RTM. However, the proposed method does not need to store plenty of wavefields, our method will be significantly faster than the traditional $Q-R T M$ with crosscorrelation imaging condition. In the future, we will continue our work in stable $Q$-RTM to solve these shortcomings.

In this paper, we just test the data with random data. In the future, we still need to analyze the sensitivity to multiples and the anti-noise property for other noises, such as the coherent noise.

\section{Conclusions}

In this paper, we propose a stable $Q$-RTM algorithm to compensate the amplitude attenuation and phase dispersion effects in the migrated images based on the excitation amplitude imaging condition. During both the forward and backward wave propagation of the stable $Q$-RTM, we use the attenuated wavefields, rather than the amplified energy of the wavefields. Thus, our Q-RTM method is relative stable and anti-noise without needing any additional processing.

We also analyze the feasibility of the excitation amplitude imaging condition, and figure out the infeasibility of the normalized crosscorrelation image condition and the instability of the crosscorrelation imaging condition for $Q$-RTM. In the backward wave propagation of the stable $Q$-RTM method, the wavefield only needs to compensate the phase dispersion. Therefore, the amplitude attenuation and phase dispersion terms of the viscoacoustic wave equation must be decoupled. Based on the decoupled viscoacoustic wave equation and excitation amplitude imaging condition, we build an efficient stable $Q$-RTM algorithm. Migration results of synthetic data show that the compensated results 
are nearly the same as those obtained by RTM from acoustic seismic data. Our method can compensate both the amplitude attenuation and phase dispersion, and improve the resolution of the migration images.

Our stable $Q$ compensated reverse time migration is based on the excitation amplitude imaging condition, whose denominator may approach zero. In this paper, we add a minimum value to the denominator of the imaging condition to control the stability of whole algorithm, but the minimum value is hard to choose. In the future, we will develop a better algorithm to solve this problem.

\section{Acknowledgments}

This work is partly supported by the Strategic Research Program of the Chinese Academy of Sciences (grant no. XDA14010303), National Natural Science Foundation of China (grant no. 41930429, 41804123).

\section{References}

[1] Aki, K., and P. Richards, 1980, Quantitative seismology, 2nd edition: University Science Books.

[2] Bickel, S. H., and R. R. Natarajan, 1985, Plane-wave q deconvolution: Geophysics, 14261439.

[3] Chen, H., H. Zhou, and S. Qu, 2014, Lowrank approximation for time domain viscoacoustic wave equation with spatially varying order fractional laplacians: International Seg Meeting, 3400-3405.

[4] Claerbout, J. F., 1971, Toward a unified theory of reflector mapping: Geophysics, 36, 467-481.

[5] Dai, N., and G. F. West, 1994, Inverse q migration: Seg Technical Program Expanded Abstracts, $1679-1684$.

[6] Deng, and McMechan, 2007, True-amplitude prestack depth migration: Geophysics, 72, S155-S166.

[7] Deng, F., 2008, Viscoelastic true-amplitude prestack reverse-time depth migration: Geophysics, 73, S143-S155.

[8] Du, Q., M. Zhang, X. Gong, and X. Chen, 2014, Polarity-consistent excitation amplitude imaging condition for elastic reverse time migration: Journal of Geophysics and Engineering, 12, 33-44(12).

[9] Dutta, G., and G. T. Schuster, 2014, Attenuation compensation for least-squares reverse time migration using the viscoacoustic-wave equation: Geophysics, 79, S251-S262.

[10] Etgen, J., S. H. Gray, and Y. Zhang, 2009, An overview of depth imaging in exploration geophysics: Geophysics, 74, WCA5-WCA17.

[11] Gu, B., Y. Liu, X. Ma, Z. Li, and G. Liang, 2015, A modified excitation amplitude imaging condition for prestack reverse time migration: Exploration Geophysics, 46, 359-370.

[12] Kaelin, B., and A. Guitton, 2006, Imaging condition for reverse time migration: Seg Technical Program Expanded Abstracts, 25, 2594-2598.

[13] Li, G., Y. Liu, H. Zheng, and W. Huang, 2015, Absorption decomposition and compensation via a two-step scheme: Geophysics, 80, V145-V155. 
[14] Li, Q., H. Zhou, Q. Zhang, H. Chen, and S. Sheng, 2016, Efficient reverse time migration based on fractional laplacian viscoacoustic wave equation: Geophysical Journal International, 204, 488-504.

[15] Mittet, R., 2007, A simple design procedure for depth extrapolation operators that compensate for absorption and dispersion: Geophysics, 72, S105.

[16] Mittet, R., R. Sollie, and K. Hokstad, 1995, Prestack depth migration with compensation for absorption: Journal of Applied Geophysics, 34, 1485-1494.

[17] Nguyen, B. D., and G. A. Mcmechan, 2013, Excitation amplitude imaging condition for prestack reverse-time migration: Geophysics, 78, S37-S46.

[18] Sun, J., T. Zhu, and S. Fomel, 2015a, Viscoacoustic modeling and imaging using low-rank approximation: Geophysics, 80, A103-A108.

[19] Sun, J., T. Zhu, J. Sun, and T. Zhu, 2015b, Stable attenuation compensation in reversetime migration: Seg Technical Program Expanded, 3942-3947.

[20] Wang, Y., 2002, A stable and efficient approach of inverse q filtering: Geophysics, 67, 657663.

[21] - 2006, Inverse q-filter for seismic resolution enhancement: Geophysics, 71, V51-V60.

[22] Yan, H., and Y. Liu, 2013, Viscoacoustic prestack reversetime migration based on the timespace domain adaptive highorder finitedifference method: Geophysical Prospecting, 61, 941954.

[23] Yu, Y., R. S. Lu, and M. M. Deal, 2002, Compensation for the effects of shallow gas attenuation with viscoacoustic wave-equation migration: Seg Technical Program Expanded Abstracts, 21, 2062-2065.

[24] Yuan, S., S. Wang, N. Tian, and Z. Wang, 2016, Stable inversion-based multitrace deabsorption method for spatial continuity preservation and weak signal compensation: Geophysics, 81, V199-V212.

[25] Zhang, J., and K. Wapenaar, 2002, Wavefield extrapolation and prestack depth migration in anelastic inhomogeneous media: Geophysical Prospecting, 50, 629-643.

[26] Zhang, Q., W. Mao, and Y. Chen, 2018, Attenuating crosstalk noise of simultaneous- source least-squares reverse time migration with gpu-based excitation amplitude imaging condition: IEEE Transactions on Geoscience and Remote Sensing, PP, 1-11.

[27] Zhang, Y., S. Xu, N. Bleistein, and G. Zhang, 2007, True-amplitude, angle-domain, commonimage gathers from one-way wave-equation migrations: Geophysics, 72, S49-S58.

[28] Zhang, Y., P. Zhang, and H. Zhang, 2010, Compensating for visco-acoustic effects in reversetime migration: Seg Technical Program Expanded Abstracts, 3160-3164.

[29] Zhou, J., and D. Wang, 2017, Vector-based excitation amplitude imaging condition for elastic rtm: Journal of Applied Geophysics, 147.

[30] Zhu, T., and J. M. Harris, 2014, Modeling acoustic wave propagation in heterogeneous attenuating media using decoupled fractional laplacians: Geophysics, 79, T105-T116.

[31] Zhu, T., J. M. Harris, and B. Biondi, 2014, Q-compensated reverse-time migration: Geophysics, 79, S77-S87. 